\title{
LX. The electric origin of molecular attraction
}

\section{William Sutherland}

To cite this article: William Sutherland (1909) LX. The electric origin of molecular attraction , Philosophical Magazine Series 6, 17:101, 657-670, DOI: 10.1080/14786440508636641

To link to this article: http://dx.doi.org/10.1080/14786440508636641

册 Published online: 21 Apr 2009.

6 Submit your article to this journal $\pi$

Џll Article views: 10

Q View related articles $\sqsubset$ 


\title{
LONDON, EDINBURGH, AND DUBLIN
}

\section{PHILOSOPHICAL MAGAZINE}

\author{
AND \\ JOURNAL OF SCIENCE.
}

[SIXTH SERIES.]

$M A Y 1909$.

\section{The Electric Origin of Molecular Attraction. By William Sutherland*}

TN connexion with this subject there is one chief outstanding difficulty to be discussed in this communication. Its nature will appear in the following few facts from the recent history of the subject. In a series of articles in this Magazine, beginning in August 1886 ([5] xxii. p. 81), I brought forward the evidence in support of the law of the inverse fourth power for molecular attraction, which may be written $3 \mathrm{~A} m_{1} m_{2} / r^{+}$in analogy with the Newtonian law of gravitation. It appeared subsequently that this analogy did not hold good in fact, that the attracting powers of molecules do not depend directly on their masses, and therefore that $3 \mathrm{~A} m_{1} m_{2}$ should be replaced by $3 a_{1} a_{2}$, in which $a_{1}$ and $a_{2}$ are parameters characteristic of the molecular masses $m_{1}$ and $m_{2}$ attracting one another. In connexion with this law of the inverse fourth power I was naturally led to give much consideration to the fact that the attraction between two small magnets similarly directed along a straight line varies inversely as the fourth power of the distance between their centres, that a similar result holds for atomic vortexes, and also for molecules containing separated electrons of opposite sign, for the significance of the electron had been emphasized in Helmholtz's Faraday lecture. But the great difficulty of connecting molecular attraction with these results for polar pairs was this, that polar pairs when oppositely

* Communicated by the Author.

Phil. Mag. S. 6. Vol. 17. No. 101. May 1909. 2 Y 
directed repel one another with a force equal to their attraction when similarly directed. According to the prevailing conceptions of the kinetic theory of gases, it seemed at that time necessary to assume that any two molecules in a gas would have their polar axes, if they possessed such, oppositely directed for as long a time as similarly directed, that therefore they would repel one another as much as they could attract, and that polar forces would not account for cohesion such as had been proved to exist in gases. Ignoring this difficulty, Fessenden in America proposed to trace cohesion to the attraction of opposite electrical charges for one another (see Phys. Review, x. 1900, with references to El. World, 1891, and Science, 1892, 1893). He seems to have obtained most encouraging results, even at these early dates, from statical considerations alone, neglecting the kinetics of molecules. 'This ignoring of kineties and of the repulsion between like charges of electricity was the probable cause of the failure of Fessenden's ideas to secure the attention of physicists. They remained almost unknown. Reinganum (Phys. Zeitschr. 1900; Ann. der Phys. [4] x. 1903) treated cohesion as of electrostatic origin, considering each molecule to have a pair of electrons at a certain distance apart. This of course gave him attractions and repulsions according to the law of the inverse fourth power. He eliminated repulsions by the consideration that by their mutual directive influence two neighbour molecules would cause one another's electric axes to be similarly directed along the line joining their centres. This is one step towards accounting for molecular attraction by means of electric bipoles, but it needs extension to show how the cohesive forces in gases are to be explained satisfactorily.

As regards gases, the main difficulty is still for the most part ignored. In solids another difficulty is possible, namely, that the distance between the centres of two neighbour molecules may be nearly equal to the distance between the two opposite electrons of a molecule. In this case the law of the inverse fourth power breaks down, and must be replaced by the more complex law for two large magnets almost in contact. This difficulty will be considered immediately. In "The Electric Origin of Molecular Attraction" (Phil Mag. [6] iv. 1902, p. 625) I sought to show that the cumulative effect of the evidence in my papers on molecular attraction between 1886 and 1902 pointed to electrical polarity as the cause of molecular attraction. To avoid the difficulty of the repulsion between molecules having their electric axes oppositely directed, especially in the case of gases, I introduced the consideration that a pair of attracting 
molecules being brought ncarer to one another by their attraction tend to increase its amount, while a pair repelling one another cause the repulsion to diminish by increase of the distance between them. There is thus a tendency for the attractive effect to preponderate. To show that the preponderance must be considerable it was argued that a pair of neighbours attracting one another would approach one another so comparatively closely as to introduce a comparatively large attraction. This principle taken with the tendency of the directive couples to promote similarity of direction in elcctric axes, was held in general to explain the preponderance of attraction, though no attempt was made to calculate definitely the amount of that preponderance. In a general way it was argued that, in reasoning about molecular ittraction, we can replace the complex medley of Nature by a representative pair of neighbour molecules having similarly directed electric axes along the line joining their centres, and therefore attracting one another.

The attractive and repulsive effects of the more remote molecules in Nature are assumed to neutralize one another. In this way I sought to give a new interpretation and use to the idea of a finite range of molecular force. The actual range of the electric forces is infinite, but their effective range is the distance between our two schematic representative neighbours having their electric axes similarly directed in the same straight line, or nearly so. The object of the present communication is to establish this conception more rigorously, and to formulate it more systematically in detail. Tn "The Pressure of Gases and the Equation of Virial" (Phil. Mag. [6] ix. 1905, p. 494) Rayleigh has investigated some of the general conditions under which the preponderance of attraction over repulsion can come to pass, and obtains strong confirmation of its reality. He considered briefly the important point as to the time during which any particular value of the virial prevails. Van der Waals, Jr., has recently attacked the problem of the law of attraction for electrical double points in the molecules of a gas (Kon. Akad. van Wet. te Amsterdam, 8 Sept. 1908, p. 132). He takes any two molecules at random and writes down the expression for their potential energy in terms of their distance apart and the directions of their axes. He then uses the law of Boltzmann to express the number of pairs of molecules which are nearly in this same relative position within a range expressed in the usual way by differentials, and then evaluates the average attraction between two molecules at distance $r$ apart, finding it to be a sum of powers of $r^{-1}$, the lowest of which is the seventh. He asserts, therefore, that the theory 
of electron pairs is excluded by the law of the inverse fourth power for molecular attraction. I think that this result about the inverse seventh power is erroneous because the writer uses in Boltzmann's law the potential energy of a pair of molecules instead of the mean potential energy of all the molecules, which is the main object of investigation. Moreover, in this case Boltzmann's law is made to give a relation between the coordinates of the two molecules, which coordinates cease to be independent variables. For these reasons I believe Boltzmann's law is made to yield a result which is not correct. Moreover, in the treatment of the problem no account is taken of the principle that attractive torces preponderate because by their own action they increase themselves, while repulsive forces diminish themselves. As a large mass of experimental evidence now furnishes cumulative support to the theory of the electric origin of molecular attraction, I shall not attempt to discuss the analysis of van der Waals, Jr., in detail, as it seems to me that the question raised by him is rather that of the correct use of the Boltzmann-Gibbs theorem than that of the origin of molecular attraction.

The ideally simplest case in which molecular force can be investigated is at the absolute zero of temperature, at which the kineties of molecules disappear, leaving the statics for unencumbered study. In "A Kinetic Theory of Solids" (Phil. Mag. [5] xxxii.) it was shown that the rigidity of metals at absolute zero could be found with considerable accuracy by a safe extrapolation from experimental data. Then it was further shown in "The Electric Origin of Rigidity and Consequences" (Phil. Mag. [6] vii.) that at absolute zero the rigidity of a collection of electrically polarized molecules is equal to their electrostatic energy per unit volume. If we imagine the electric polarization to consist in each molecule's having an electron pair $b=$ whose charges are $e$ at distance $\left(m / \mu_{0}\right)^{1 / 3}$ apart, $m$ being the mas* of the molecule and $\rho_{0}$ the density of the metal, with $\mathrm{K}$ for its dielectric capacity and $\mathrm{N}$ for the rigidity at absolute zero, then this result is given by the formula

$$
\mathrm{N}=\frac{2 \pi\left(m / \rho_{0}\right)^{2.3}}{3 \mathrm{~K}\left(m / \rho_{0}\right)^{2}}, \quad \cdot \quad \cdot \quad \cdot .
$$

which was found to express the experimentally derived facts satisfactorily. This is simply Maxwell's expression $2 \pi \mathrm{D}^{2} / \mathrm{K}$ for the energy in a dielectric associated with an electric displacement $\mathbf{D}$, for, when we consider the electric displacement per unit area corresponding with $e$ per molecule, we get $e /\left(m / \rho_{0}\right)^{2 / 3}$ to be nsed instead of $D$, the result being divided by 3 for statistical reasons.

But we must look more closely into the differences between 
a natural collection of molecules and the dielectric in an electric field. The main one is that in the electric field there is a resultant direction of electric force, whereas in a collection of molecules there is none, the electric force inside a molecule being reversed between the opposite poles of two molecules. The very definite conception which reduces the polarity of a molecule to two point electrons at the ends of a diameter of the molecule's supposedly spherical surface, becomes almost a hindrance in the case of molecules in contact at absolute zero because of the tendency of two oppositely charged point electrons to come nearly into coincidence and nearly to destroy one another's effect. In the facts of Nature there is no indication of any tendency to such destruction of effect. We shall do better then to regard the molecule as polarized throughout its mass, just in the same way as the Earth is magnetically polarized, and can be replaced by an infinitely short magnet at its centre, if only the magnetic moment of this is equal to that of the Earth. Just as we should encounter difficulties if we treated the Earth as a sphere having magnetic poles at the ends of a diameter, we are confronted with similar ones if we treat the molecule as an electric bipole.

With bipoles in which the axis between the poles is a diameter or nearly a diameter, the law of the inverse fourth power would break down completely, as it holds only for bipoles in which the axis is small compared with the distance between the attracting bipoles. But if the molecule is taken to be a uniformly electrically polarized sphere, which may be called a uniformly electrized sphere, then its external effect is the same as that of an infinitely short bipole at its centre, the bipole having the same electric moment as the sphere. With uniformly electrized spheres having their axes similarly directed along the line joining their centres, the law of the inverse fourth power becomes exactly correct at all distances. I hope to show in a separate communication that the conception of a uniformly electrized molecule is helpful in studying the vibrations causing spectra.

As I have shown (Phil. Mag. [6] iv. p. 636) that for many molecules the electric moment $e\left(m / \rho_{0}\right)^{1 / 3}$ is actually nearly equal to the product of electron charge and molecular diameter, the uniform electrization of the molecule is intimately related with the electron charge $e$ of electrolysis. When a number of uniformly electrized spheres are brought into contact at absolute zero, we get a mass which is uniformly electrized, but with the axis of electrization changing in direction so that the average electric force throughout the mass is nil. To get a schematic representation of such a state of affairs, 
in "The Electric Origin of Rigidity and Consequenees" I used the artifice of dividing the space into cubes each containing a molecule, and then imagining the electrization of the mass caused by alternate sheets of positive and negative electricity in the planes diviling the space into cubes, so that each of the three plane distributions bad a third part of the potential energy of the whole mass. Another method of representation is to place negative electrons $b$ and positive alternately at the corners of the cubes so that along any line forming edges $b$ and $\$$ recur alternately. If with $b$ of amount $e$ as origin we draw three rectangular axes forming the edges of the eight enbes which meet at $b$ and place $\#$ of anount $e$ at distances $s$ and $-s$ on each axis, we see that our arrangement provides for a large preponderance of attraction over repulsion, since the six nearest neighbours of $b$ are - It provides a distribution of polarity similar to that in our mass of uniformly electrized molecules. I have sought to

Fig. 1.

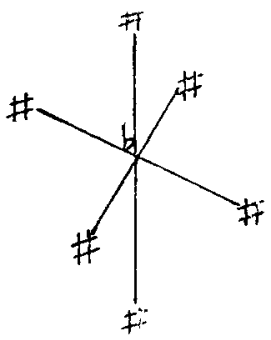
show in "Ionization in Solutions and Two New Types of Viscosity" (Phil. Mag. [6] xiv. 1907, p. 1) that the ions of solutions give such a distribution of polarity as that just described. An expression of the same form as (1) for the rigidity of such a distribution of electrons leads to correct results concerning the electric resistance of electrolytic solutions. Since the form (1) applies to metals at absolute zero and to uniformly spaced ions, it follows that a cubical arrangement of electrons is for some purposes a convenient and proper simplified represcntation of the electric polarity in a metal at absolute zero. But this leads to a scheme which is better for most purposes because liker to the natural. Imagine a mass of molecules at absolute zero to consist of cubes represented in plan in the figure where the arrow at the centre of each square denotes the direction of the uniform electrization of the cube. Here the cubes along $A D$ and parallel to it form prisms which are alternately electrized in opposite directions. The next row of

Fig. 2.

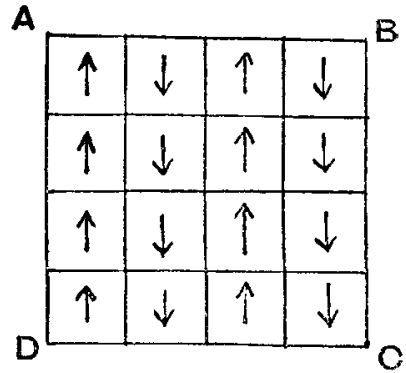
cubes above $A D$ would form a prism oppositely electrized 
to AD. If we call the direction of $\mathrm{AD}$ axial and directions at right angles lateral, we can say that a cube is electrized in the same direction as its two nearest axial neighbours and in a direction opposite to that of its four nearest lateral neighbours. Each cube attracts its six nearest neighbours. I believe that this constitutes the chief part of the solution of the main problem of cohesion. Consider two cubes in lateral relation and electrized in the same direction. They will repel one another till they reach the position of minimum potential energy when infinitely apart. But two cubes in lateral relation electrized in opposite directions will attract one another to the position of minimum potential energy when they are in contact. For a relative position between axial and lateral the force between two cubes vanishes. We conclude, then, that the principle of minimum potential energy in Nature acts so as to give such an arrangement as is set forth schematically in the last figure. In cubical crystals the scheme may be a still closer representation of fact. In the case of an amorphous mixture of substances whose molecules are of different size and shape, we can make a diagram of the state of affairs, such as that here given. Let $\mathrm{AD}$ and $\mathrm{BC}$ be two lines representing the prevailing

Fig. 3.

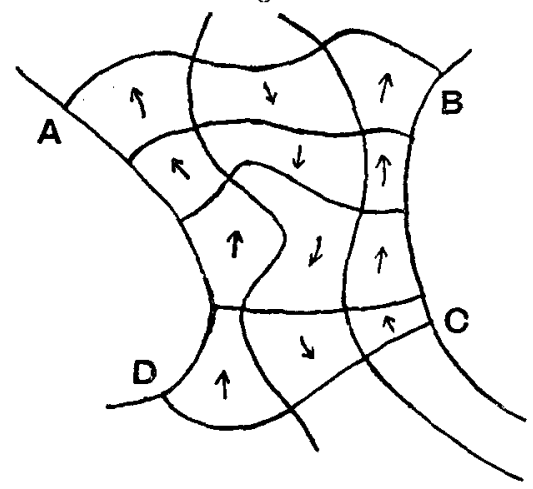
direction of electrization. They correspond with the straight lines $A D$ and $\mathrm{BC}$ of fig. 2. They are like lines of electric force. Between them lie two similar curved lines. Then draw the system of lines that cut these orthogonally like equipotential surface. These two sets of lines divide up the space into regions corresponding with the squares of fig. 2, each region representing the domain of a molecule. The arrow indicates the direction of electrization of each molecule. Here we have the conditions for cohesion in an amorphons substance, the figure showing how in a body of finite size there may be no prevailing direction of electrization. Here we are reminded of the theoretically infinite range of the force of each molecule and its practical restriction almost to the nearest neighbours. 
But a most interesting problem arises when we extend these ideas from a solid at absolute zero to a gas at ordinary temperatures. The gas still shows cohesion, but how are we to imagine the axes of electrization of neighbour molecules in a gas to be related in a manner similar to that shown in figs. 2 and 3 . Fessenden assumed that in a gas the molecules behave as if each molecule has an electric charge opposite to that of its immediate neighbours, and so he proposed to replace the equation of van der Waals $\left(p+a / v^{2}\right)(v-b)=\mathrm{RT}$ by a form in which the cohesional term $a / v^{2}$ is replaced by $a / v^{4 / 3}$. I do not know of any experimental evidence that he ever submitted in support of this change. In "The Laws of Molecular Force" (Phil. Mag. [5] xxxv. 1893) I have shown that the equation of van der Waals applies to the element gases and methane, but not to compound gases in general, for which the cohesional virial tends to the form $l /(v+k)$ instead of $l / v$ or the $a / v$ of van der Waals. Now although it has just been proved that Fessenden's assumption is a convenient and proper simplification for a number of uniformly electrized molecules in contact, there is no warrant for it in the case of gases when we are studying the mutual potential energy of molecules. We shall see it apply to total potential energy. It does apply to the ions of a solution as the electrical evidence shows.

Since gases do not behave as completely ionized substances, the electrical evidence is also directly opposed to the literal truth of Fessenden's assumption for gases. As a fiction it has no schematic convenience in the study of characteristic equations, because it implies that the uniformly electrized sphorical molecule of a gas converts the whole of its domain into a uniformly electrized sphere. We shall see that the whole potential energy, not merely the mutual, is that of a uniformly electrized spherical domain. Yet although there is no theoretical or experimental justification of the literal truth of Fessenden's assumption for gases, there has been discovered by J. E. Mills a remarkable relation which seems to verify it completely. This paradox contains matter of importance for the whole of molecular physics, and the rest of the present communication will be devoted to an attempt to elucidate it. This is the relation of Mills : Let $\mathrm{L}$ be the latent heat in calories for the evaporation of a liquid of density $\rho=d$ at temperature $\mathrm{T}$ to the state of saturated vapour of density $\rho=\mathrm{D}$ at $\mathrm{T}$, and let $\mathrm{E}$ be the thermal equivalent of the work done in changing the density from $d$ to $\mathrm{D}$ against the saturation pressure; so that $\mathrm{L}-\mathrm{E}$ is the 
internal latent heat, then at all temperatures up to the critical

$$
\frac{\mathrm{L}-\mathrm{E}}{d^{1 / 3}-\mathrm{D}^{1 / 3}}=\text { constant characteristic of liquid. . }
$$

This has been verified by a most exhaustive examination of the data for 31 liquids of diverse chemical types, compounds but not elements (Journal of Physical Chemistry, vi. 1902 , p. 209 , viii. 1904 , pp. 383,593 , ix. 1905 , p. 402, x. 1906, p. 1). Mills interprets his relation by treating cohesion as due to gravitation, taking $d^{1 / 3}-D^{1 / 3}$ as a measure of the change of the mutual gravitational potential energy of the molecules when they pass from the state of density $d$ to that of density D. There are two well-known and perfectly definite reasons why gravitation cannot account for cohesion: first, the forces between neighbour molecules are enormously greater than their mutual gravitation; and, second, the latent heat required to evaporate molecules against their gravitation varies for unit mass with the size of the mass evaporated. This variation has never been discovered because the latent heat of evaporation against gravitation is so minute a fraction of the latent heat of evaporation against cohesional force, that its variations are beyond the reach of existing experimental refinements to detect. If Mills had traced his relation to the law of the inverse square as it operates in a uniform mixture of equal numbers of equal opposite charges of electricity, he would have been on the right track with Fessenden. In this case, the repulsions between the like charges introduce an element unlike gravitation. This mixture of electric charges has been shown above to be a convenient and proper schematic representation sometimes for a collection of uniformly electrized molecules in contact. Let us waive the stipulation about the molecules being in contact at absolute zero, and let us take (1) for the electrostatic energy of the molecules in unit volume of a liquid of density $\rho$ or $d$. Then for a molecule of volume $m / d$ the electrostatic energy will be $2 \pi / 3 \mathrm{~K}(m / d)^{1 / 3}$. If with Fessenden we assume that exactly a similar condition of polarity prevails in the saturated vapour of density $D$, we can write $2 \pi \mathrm{D}^{1 / 3} / 3 \mathrm{~K} \mathrm{~m}^{1 / 3}$ for the electrostatic energy of the vapour, assuming for the moment that $K$ does not change. The change is $2 \pi\left(d^{1 / 3}-\mathrm{D}^{1 / 3}\right) / 3 \mathrm{~K} \mathrm{~m}^{1 / 3}$. If this is equivalent to the internal latent heat, then for a given substance

$$
\frac{\mathrm{L}-\mathrm{E}}{d^{1 / 3}-\mathrm{D}^{1 / 3}}=\text { constant at all temperatures. . . }
$$

This is the result which appears to justify Fessenden's 
treatment of a gas as a completely ionized fluid, and is one by which Mills might have given a dynamical interpretation of his discovery, instead of connecting it with gravitation. A notable fact in this relation is the disappearance of $K$, because in assuming $K$ constant during the change of state from liquid to vapour, we have virtually made it disappear. Now in the laws of the parameter $a$ in $3 a_{1} a_{2} / r^{4}$ for molecular attraction in my rarious Phil. Mag. papers on this subject, it has never been necessary or advantageous to consider $K$ as exercising any influence; it disappears from the scene. These two instances of the disappearance of $\mathrm{K}$ are most easily accounted for by the hypothesis that each molecule behaves as an electrized sphere. The external mutual effect of two such neighbour spheres depends only upon the electric moment assigned to each, just as the mutual effect of two neighbour magnetized spheres depends only upon the magnetic moment of each, and not upon the permeability of the matter of the spheres, if mutual induiction is not operative. Thus in (1) $\mathrm{K}$ may be allowed to disappear by being merged in $e^{2}\left(\mathrm{~m} / \rho_{0}\right)^{2 / 3}$, the square of the electric moment of a molecule. Let us denote the electric moment by es and replace (1) by

$$
\mathrm{N}=\frac{2 \pi}{3}\left(\frac{e s}{m / \rho}\right)^{2} \ldots . \quad . \quad . \quad .
$$

This discarding of $K$ or this putting it equal to 1 expresses definitely the principle that cohesion is the attraction of immediate neighbours through the ether. In the theory of ions in solution $K$ plays a prominent part, because neighbour ions are separated by the solvent of dielectric capacity $K$.

This equation (4) gives the electrostatic energy per unit volume: that per molecule is $2 \pi e^{2} s^{2} / 3(\mathrm{~m} / \mathrm{\rho})$. If now $s$ were equal to $(m / d)^{1 / 3}$ in the liquid and to $(m / D)^{1 / 3}$ in the vapour, we should obtain at once the formula of Mills. The most important point to understand then is this: How does the molecule of vapour behave as if its uniform electrization extends not only through its rolume $m / \rho$ or $m / d$, but through its domain $m / \mathrm{D}$ ? The answer is to be found by considering the electrostatic energy of a molecule in two parts, the intermolecular and the intramolecular, or the mutual and the self energy. For many purposes it is convenient with Maxwell to localize so much electric or magnetic energy in a given element of volume. Thus in our collection of molecules at absolute zcro we assign electrostatic energy $-2 \pi e^{2} s^{2} / 3(\mathrm{~m} / \mathrm{\rho})$ to each molecule, though we may drop the negative sign, as above, when it is not essential. But this 
energy must be largely a mutual affair, and its localization in a molecule is only a convenience and not a necessity. When we expand the collection of molecules to density D in such a way that each always attracts its six nearest neighbours through the electrization, the mutual potential energy per molecule is proportional to $-e^{2} s^{2} /(m / D)$ and may be written $-2 \pi e^{2} s^{2} / 3(m / D)$. If the total potential energy is $-2 \pi e^{2} / 3(\mathrm{~m} / \mathrm{D})^{\frac{1}{3}}$, then the internal or self-energy is

$$
-\frac{2 \pi e^{2}}{3}\left\{\left(\frac{\mathrm{D}}{m}\right)^{\frac{1}{3}}-\frac{\mathrm{D}}{m} s^{2}\right\} . \cdot \cdot \cdot
$$

Thus, then, for any density $\rho$ we divide the potential energy per molecule up into two parts, the mutual $-2 \pi e^{2} s^{2} / 3(m / \rho)$, and the internal $-2 \pi e^{2}\left\{(\rho / m)^{1 / 3}-s^{2}(\rho / m)\right\} / 3$. At absolute zero this scheme makes the internal energy nil, the whole potential energy being mutual of amount $-2 \pi e^{-2} k^{2} / 3\left(m / \rho_{0}\right)$. The total electrostatic energy $-2 \pi e^{2}(\rho / m)^{1 / 3} / 3$ does not appear in the equation of the virial or in the corresponding characteristic equation of gases, liquids, and solids, wherein the mutual relations of molecules are discussed. In these characteristic equations the mutual potential energy $-2 \pi e^{2} s^{2} \rho / 3 m$ appears by itself or in association with other expressions for mutual potential energy during molecular collisions. The discovery of Mills may be stated as the following principle:The total potential energy of a number of like molecules is the same as if each caused its own domain to be uniformly electrized. with an electric moment proportional to the linear dimension of the domain, the direction of electrization being such that in general any molecule attracts its six immediate neighbours. Once again we are face to face with the difficulty in a gas of satisfying the condition that a molecule and its six immediate neighbours may have their electric axes related as in figs. 2 and 3 . We seem to be led to the conclusion that in the molecules of a gas the electric axes change their direction with sufficient rapidity and in such a manner that as nearly as possible each molecule is attracting its six immediate neighbours. Thus we can apply fig. 3 to a gas, each of the four-sided regions corresponding with a six-faced region of space forming the domain of a gaseous molecule, the molecule being centrally situated. In this way then we trace cohesion in a gas to electric polarity, avoiding the difficulty of repulsions by the consideration that under a sort of mutual induction between neighbours and in accordance with the principle of minimum potential energy the polarities of neighbour molecules are related to one another according to the scheme of figs. 2 and 3 . The same explanation applies 
to the case of liquids and solids with only this difference, that the electric axis of a molecule may not change its position in the molecule so frequently through a large angle as in the case of a gas where the free motion causes numerous and rapid changes of neighbours.

In the further development of this conception interest centres round the laws of $e s$, the electric moment of a molecule. These have been investigated in several of my Phil. Mag. papers on molecular attraction. In a broad way we can divide chemical compounds into two classes, those which are ionized in solution and those which are not. To the former belong the great majority of organic compounds and the compounds of the non-metals with one another. Typical of the latter are the salts of the metals. In both classes of compounds $e s$ for a molecule can be derived from values of es belonging to ench atom. In those compounds which are not ionized in solution there is an approximation to the following simple rule, that in each atom $e s$ is proportional to the volume of the atom, and therefore that es for the molecule is proportional to the volume of the molecule. But as es divided by the volume of the molecule gives the intensity of electrization or the electric polarization per unit area, we have the simple approximate result that in this class of compounds the intensity of the electrization of molecules is an absolute constant. This result shows that for a detailed investigation of the structure of atoms, especially in connexion with the electron theory of matter, it will be necessary to carry out more extended studies of es or of $\left(\mathrm{M}^{2} l\right)^{1 / 2}$, as a proportional quantity is denoted in some of my papers.

\section{Summary.}

The electric polarity of molecule or atom may be represented as caused in two ways, either by the separation of two electrons $e$ of opposite sign throngh a distance $s$ giving an electron pair or bipole of moment $e s$, or by considering the molecule or atom to be uniformly or variably electrized so as to have a total electric moment es. In the same way, the Earth as regards external magnetic effects may be treated either as a uniformly magnetized sphere or as a pair of opposite magnetic poles near the centre and having a magnetic moment equal to that of the Earth. At present we may regard the atom as electrized in both ways to account for the electron of electrolysis and for the electron structure of matter. Doubtless the two ways are but different aspects of one phenomenon, and for many purposes we can regard the resultant electric polarity of an atom as being due purely 
to its electrization. As each atom is equivalent to an infinitely short bipole of equal moment at its centre, the law becomes rigorous that one atom acts on any other with a force inversely proportional to the fourth power of the distance between their centres. If the relative directions of the two electric axes of two atoms are random, repulsion will occur as frequently as attraction. This is the great difficulty in accounting for cohesion by means of electric polarity of atoms and molecules, especially in the case of gases in which the kinetic theory has made random distribution the ideal of generality. It was previously pointed out that as the attractive forces tend to increase themselves, while the repulsive tend by their own action to diminish themselves, there must be a tendency even in a random distribution of polarities for the attractive forces to preponderate. But it is now suggested that the electrization of neighbour molecules is not randomly direeted, but is so directed that an atom is attracted by each of its six nearest neighbours. This satisfies the main condition for minimum electric potential energy in a set of moving molecules whose axes of electrization are free from constraint except that of being translated with the molecule. Beyond the range of the six nearest neighbours the attractions and repulsions of a molecule tend to become more nearly equal and opposite the greater the distance. Thus we bave a range of force which is actually infinite but is effectively an attraction reaching only the six nearest neighbours of a molecule. The effective range of molecular.attraction is the distance between a molecule and its immediate neighbours. This principle is verified by the fact that dielectric capacity does not appear in values of cohesion. Although in a small group of molecules, say a molecule and its six nearest neighbours, there is at any instant a prevailing direction of electrization, this varies with time, and at a given instant of time varies gradually from one group to the next, so that throughout a large number of molecules the directions of electrization are as many as if distributed at random. The electrostatic energy $\mathrm{N}$ per unit volume of a collection of such molecules of mass $m$ and density $\rho_{0}$ in contact at absolute zero is $-2 \pi e^{2} / 3\left(m / \rho_{0}\right)^{1 / 3}$, and the energy per molecule is $-2 \pi e^{2} / 2\left(\mathrm{~m} / \rho_{0}\right)^{1 / 3}$, the dielectric capacity $K$ not appearing. But Mills has discovered that the internal latent heat of evaporation of a liquid being $\mathrm{L}-\mathrm{E}$ at any temperature at which the densities of liquid and saturated vapour are $\rho=d$ and $\rho=\mathrm{D},(\mathrm{L}-\mathrm{E}) /\left(d^{1 / 3}-\mathrm{D}^{1 / 3}\right)$ is independent of temperature. This can be accounted for most readily by generalizing this last formula and making the 
total electrostatic energy of a molecule at any density $\rho$ equal to $-2 \pi e^{2} / 3(m / \rho)^{1 / 3}$. But according to the law of the inverse fourth power the mutual potential energy of the attracting molecules is $-2 \pi e^{2} s^{2} / 3(\mathrm{~m} / \rho)$ per molecule. It appears then that $-2 \pi e^{2}\left\{(\mathrm{~m} / \rho)^{2 / 3}-s^{2}\right\} / 3(\mathrm{~m} / \rho)$ must be internal electrostatic energy per molecule. The total electrostatic energy of a number of molecules is of the same form as that of equal numbers of positive and negative electric charges uniformly mixed as in the ions of an electrolytic solution. Thus an hypothesis advanced years ago by Fessenden in America seems, when suitably interpreted, to be justified by the discovery of Mills made also in America, though both ignored fundamental physical difficulties in obtaining their results. To account for the facts it is proposed to look upon atoms as electrized, just as we speak of a magnet as being magnetized. But an electrized molecule causes the whole of its domain to be electrized as if for a given chemical substance each molecule possessed an invariable amount of positive electricity and an equal amount of negative, and as if these amounts were uniformly distributed through the domain of the molecule, whether its state is gaseous, liquid, or solid. This statement applies only to considerations respecting the total potential energy of molecules. In other words, as regards total energy each molecule behaves as if it had an electric moment proportional to the linear dimensions: of its domain, whereas in the matter of mutual energy each molecule has an electric moment of amount es investigated in connexion with the laws of molecular attraction.

Melbourne, Feb. 1909.

LXI. A Note on the Production of Steady Electric Oscillations in Closed Circuits and a Method of Testing Radiotelegraphie: Receivers. By J. A. Fleming, M.A., D.Sc., F.R.S., and G. B. Dyкe, $B . S c \cdot *$

IN testing radiotelegraphic detectors the difficulty is 1 generally to obtain facilities for working in actual stations and at various distances. Thus, if an inventor desires to know whether an improvement which he has made in oscillation detectors is an advance on anything yet done, he must be able to test this receiver at a station in correspondence with others at various and at considerable distances, and even then quantitative measurements are difficult, or impossible, to obtain on account of the continually varying atmospheric conditions which, as is well known, introdnce

* Communicated by the Physical Society: read March 26, 1909. 